International Journal of Applied Dental Sciences 2021; 7(4): 162-165

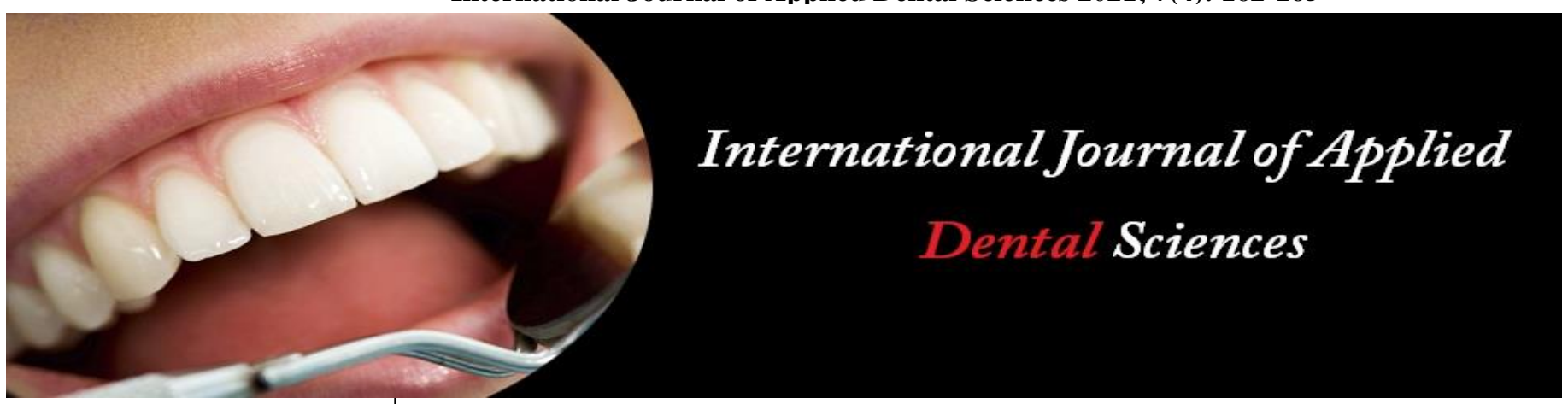

ISSN Print: 2394-7489

ISSN Online: 2394-7497

IJADS 2021; 7(4): 162-165

(C) 2021 IJADS

www.oraljournal.com

Received: 13-08-2021

Accepted: 15-09-2021

Puja C Yavagal

Professor, Department of Public

Health Dentistry, Bapuji Dental

College and Hospital,

Davanagere, Karnataka, India

Manjusha Umbranikar

Postgraduate Student,

Department of Public Health

Dentistry, Bapuji Dental College

and Hospital, Davanagere,

Karnataka, India

Gazala Sulthana DS

Postgraduate Student,

Department of Public Health

Dentistry, Bapuji Dental College

and Hospital, Davanagere,

Karnataka, India

\section{Applications of digital technology in dental public health: A narrative review}

\section{Puja C Yavagal, Manjusha Umbranikar and Gazala Sulthana DS}

DOI: $\underline{\text { https://doi.org/10.22271/oral.2021.v7.i4c.1366 }}$

\section{Abstract}

Digital technologies in dental public health are being used in a variety of ways to improve healthcare, reduce health care costs, and improve performance of public health programs. This narrative review summarises the important applications of digital technologies in the field of dental public health and initiatives of Government of India towards digitization of public health system. Literature review through various databases like Web of Science, Pub Med, Scopus, Cinahl, PUBMED, Google Scholar, Embase, ScienceDirect, Directory of Open Access Journals was done. Results of review highlight the effective use of digital technology in the development of electronic health records (EHRs); personalised medicine and health data linkage; clinical decision support for novel treatment concepts and deep learning and Artificial Intelligence (AI) for diagnostic analysis. Adoption of mobile technologies in the dental field is fundamentally transforming how dental healthcare is viewed, given, and consumed. However, lack of data security and protection laws, technical infrastructure, advanced biological sensors, orientation among public health workers, linguistic diversity, patients' fear and unfamiliarity makes the application of digital technology in dental public health practice challenging.

Keywords: dental public health, digital dentistry, e-dant seva

\section{Introduction}

Dental care has become unaffordable for many individuals as a result of economic crisis and its consequences on healthcare systems, and a large number of patients do not seek for much needed dental treatments. Along with this, ageing of the global population, as well as the resulting growth in general and dental care needs, raises worries about the long-term viability of healthcare systems. These changes underline the urgent need for a new dental care model that is both sustainable and efficient ${ }^{[1]}$. In this context, the most promising strategy for transforming the oral health care is the adoption of digital technology. The landscape of dental public health is being reshaped by digital oral health care. It refers to the use of dental technologies or gadgets to deliver dental treatment that include digital or computer-controlled components. World Health Organization (WHO) advocates and encourages the creation and spread of public health practises that are supported by both information and communication technology (e-Health) and mobile devices (m-Health) ${ }^{[2]}$. Dental public health focuses on patient centred care model and the use of digital technology in dentistry provides an opportunity to create a shift from a "disease-centred" to a "patient-centred" dental care. Digital science has permeated into different facets of dental public health.

\section{Review}

2.1 Digital Imaging in Oral Epidemiology: "A cutting edge science"

In oral epidemiology, digital imaging has been used to circumvent some of the constraints of direct clinical assessment. Recent research has shown that observer bias can be successfully mitigated by using digital imaging which allows to remotely score anonymized images permitting effective examiner blinding. Simultaneous assessment by multiple examiners across multiple locations, reduces training/calibration time and permits standardisation of examination conditions ${ }^{[3]}$. Archived images improve data storage, research governance, reassessment of conditions using different indices, as well as robust follow-up assessment ${ }^{[3]}$.
Corresponding Author: Puja C Yavagal

Professor, Department of Public Health Dentistry, Bapuji Dental College and Hospital,

Davanagere, Karnataka, India 
Dental auxiliary team like nurses and hygienists could be trained to capture images, reducing the logistical complexities and cost of labour and consumables associated with mobile examination units. Contrast optimisation, noise reduction, colour standardisation, and artefact reduction are some of the processing strategies that can improve the diagnostic performance and variable quality of digital photographs ${ }^{[4]}$. However, the loss of tactile feedback and an inability to assess oral structures from different positions using different lighting conditions results in a loss of holistic clinical judgement. The cost of developing or purchasing suitable equipment as well as provision of satisfactory training in its use can be a barrier to implement dental imaging in public health practice ${ }^{[4]}$.

\subsection{Digital Risk Assessment models and screening tests: "Nipping the evil in the bud"}

Electronic oral health screening system (EOHSS) uses images for the detection and staging of oral conditions. It is designed for use in field conditions operated by a dentist, dental hygienist, or other suitably trained individuals. Digital Caries risk prediction models which have been effectively used in populations include: CAMBRA (caries management by risk assessment); Cariogram and CRAFT (Caries risk assessment for treatment) ${ }^{[5]}$. Periodontal disease risk assessment models like Periodontal Risk Calculator (PRC); Risk AssessmentBased Individualized Treatment (RABIT); Genetic tests have provided new dimension for screening and mapping periodontal of disease. ${ }^{6}$ Dual Camera systems with novel software algorithms, are used to classify and map fluorosis in communities ${ }^{[3]}$. Convolutional Neural Network (CNN) digital models are used to detect and localize malocclusions ${ }^{[7]}$. Salivaomics, saliva-based digital techniques for early diagnosis of dental diseases and identification of biomarkers of potential oral and systemic diseases, is a boon for dental public health ${ }^{[8]}$. Digital oral cancer screening tests coupled with Artificial Neural Network (ANN) based riskstratification are valuable Point-of-Care (PoC) tools for early detection/screening of oral potentially malignant lesions. ${ }^{9}$ Tele-cytology system has raised the bar of cancer screening in India. Few of the effectively used devices include VELscope and Oral Scan ${ }^{[10]}$.

\subsection{Initiatives of Government of India: "Ray of Hope"}

Ayushman Bharath noncommunicable diseases programme involves Digital multiphasic oral cancer screening, where, community health workers are issued tablets with preloaded screening algorithms designed to collect and record data, and making referrals ${ }^{[11]}$. Extension of Community Outcomes project incorporates hub-and-spokes model, where, experts (the hub) provide a virtual training course for community health-care workers (the spokes) for screening oral diseases ${ }^{[12]}$. NITI Aayog, is launching a project for, national digital repository of annotated and curated cancer pathology images ${ }^{[12]}$. Imaging biobank for cancer project (IBCP), is expected to provide an unprecedented opportunity for use of artificial intelligence to inform decision-making in cancer treatment at low cost ${ }^{[13]}$. The National Health Policy (NHP) (2017) has set goals for the adoption of digital technology in healthcare. These include developing district-level electronic databases, interoperable electronic health records, and registries for all diseases of public concern including oral diseases, as well as linking systems across health providers at the state and national levels using Metadata and Data Standards (MDDS) for seamless data and service sharing, and envisioning the health-tech ecosystem as a Federated National Health Information Architecture. It covers various sectors like: Digital Health Ecosystem; Health Information System (HIS); medical education and development of health information database. In order to introduce a uniform standardised system for creation and maintenance of health records by healthcare providers, the Electronic Health Records Standards were updated in 2016 by the Ministry of Health and Family Welfare ${ }^{[12]}$. The National electronic Health Authority (NeHA) was set up in 2015 under the MoHFW. NeHA acts as an enforcement body and is responsible for the enforcement of standards and ensuring security, confidentiality, and privacy of patient's health information and records. Ministry of Health and Family Welfare has released the "National Digital Health Blueprint" (NDHB) in 2019. It provides an action plan for realising Digital Public Health ${ }^{[12]}$. The National Strategy for Artificial Intelligence launched in June 2018 focuses on how India can leverage the transformative technologies like digital technology to achieve social and equitable growth. The focus is on the potential of artificial intelligence combined with robotics and Internet of Medical Things (IoMT) to address healthcare problems through research and training, early detection, diagnostic, decision making and treatment. The Ministry of Electronics and Information Technology (MeitY), which is the nodal ministry in-charge of the "Digital India" programme, works in conjunction with the MoHFW and NITI Aayog to ensure digitalisation of public health care in India ${ }^{[12]}$.

\subsection{Digital health records: "A systematic and sustainable data collection approach"}

Unlike paper-based systems, the use of electronic health record encourages adherence to documentation standards by forcing dentists to enter data in a structured format, allows for monitoring and encourages information sharing among various members of the healthcare team. Nonetheless, the finest data algorithms are only as good as the original input's trustworthiness and validity [14]. Poor data quality characterised by missing and incomplete data, is a major issue in healthcare data collecting. This arises when there is no standardised format for gathering data or when certain population cohorts' data is not adequately captured. For valuebased healthcare, better service and improved health outcomes at a lesser cost, uniform data standards in terminology, diagnosis and treatments, and software applications are critical. The use of standardised forms and templates to limit the chance of missing or incomplete data would considerably aid data consistency and later aggregation for analysis, learning, and quality improvement ${ }^{[14]}$. Digital population health records linked at the personalized individual level of information and data is a great model for the health economic policy. The health data can be tagged to their sociodemographic profile and linked to national identifying profile like Aadhar card. This may help in disease prediction and prognostics modelling, designing preventive programs, public health research, planning programs, governance and delivery of the oral healthcare system. Digital records serve as an excellent link between the dentist, dental auxiliary team, community health workers and physicians to understand, collaborate, and deliver an optimum level of oral healthcare.

\subsection{Digital Public health informatics: "The contemporary facet of Dental Public Health"}

The application of informatics in the areas of public health surveillance, prevention, preparedness, and health promotion 
is known as public health informatics. The main purpose of dental informatics is to enhance patient outcomes and make dental treatment more competently delivered. Public health dentists can solve more clinical problems by supporting and improving prevention, diagnosis, treatment, with strong evidence-based practises by maintaining or enhancing costbenefit ratios for oral health ${ }^{[15]}$. Biostatistics, tele health education, Geospatial Information System (GIS) and tele dentistry are the main areas where dental informatics is applied ${ }^{[16]}$. Research and biostatistics: Big data analysis using digital records is a big boost for epidemiological research. Software programs like SPSS (Statistical Package for the Social Sciences), SAS (Statistical Analysis System) and EPIINFO have made big data analysis easy and simple [17]. Epicollect software allows to submit geotagged data forms to a central health project website from mobile phones ${ }^{[18]}$. Tele Community Health Education: Software programs for oral health education are available at m-Health: National Health Portal of India and e-Dantseva websites which may be utilized at health centres for creating oral health awareness ${ }^{[19]}$. Cloud computing is a promising area for mass health education and training of health workers to create oral health awareness ${ }^{[20]}$. Geographic Information System (GIS) involves computer-based tools which geomap health data thereby helping in disease surveillance, analysing health trends, determining risk factors, assessing resource allocation; planning and targeting interventions. Software components used in GIS include: (a) a Database Management System (DBMS) (b) tools for the entry and modification of geographic data (c) tools that facilitate geographic query, analysis, and visualisation d) tools for creating a graphical user interface (GUI) ${ }^{[21]}$.

\subsection{Tele dentistry and m-Health: "Improving access to oral health care"}

m-Health: National Health Portal of India App is a mobile application which acts as a mobile extension to the National Health Portal of the Government of India (GoI). The app features an intuitive UI that makes the access and discovery of health information easy for the rural audience who are predominantly illiterate or semi-literate, which are deployed in the panchayats connected by broadband. Collaborative Digital Diagnosis System (CollabDDS) is developed for tele-consultation, diagnosis, remote education and as a data repository. It is a remote expert dental programme introduced by Centre for Dental Education and Research at the All-India Institute of Medical Sciences in New Delhi. ${ }^{22}$ Health Management Information System (HMIS) software is developed, by AIIMS, where information recorded (input) is stored and retrieved for public health decision-making (output) ${ }^{[23]}$.

\section{Challenges for Digitalization of Dental Public Health Care System}

Some of the major road blocks in incorporation of digital technology in dental public health practice are; data security and protection laws, technical infrastructure, advanced biological sensors, bandwidth support, orientation among public health workers, linguistic diversity, along with patients' fear and unfamiliarity.

\section{Conclusion}

Digital innovations for improving oral health of community prove effectiveness in terms of saving cost and time, improves accuracy of public health interventions provides a high level of predictability of outcomes thus is a major step towards bridging inequality in oral health care between rural and urban communities.

\section{References}

1. Shetty V, Yamamoto J, Yale K. Re-architecting oral healthcare for the 21st century. J. Dent 2018;74(1):S10S14.

2. Neville P, van der Zande MM. Dentistry, e-health and digitalisation: A critical narrative review of the dental literature on digital technologies with insights from health and technology studies. Community Dent Health. 2020;37:51-58.

3. Hogan R, Goodwin M, Boothman N, Iafolla T, Pretty IA. Further opportunities for digital imaging in dental epidemiology. Journal of Dentistry 2018;74: S2-9.

4. Hill H, Birch S, Tickle M, McDonald R, Brocklehurst P. The technical efficiency of oral healthcare provision: Evaluating role substitution in National Health Service dental practices in England. Community Dent Oral Epidemiol 2017;45(4):310-316.

5. Su N, Lagerweij MD, van der Heijden GJ. Assessment of predictive performance of caries risk assessment models based on a systematic review and meta-analysis. Journal of Dentistry 2021;110:103664.

6. Eshwar S, Ankola AV, Kumar A, Hebbal M. Evaluation of periodontal risk assessment model among adults aged 30-60 years attending KLE Dental College, Belgaum: A hospital-based study. J Indian Soc Periodontol 2010;14(3):173-7.

7. Talaat S, Kaboudan A, Talaat W, Kusnoto B, Sanchez F, Elnagar MH et al. The validity of an artificial intelligence application for assessment of orthodontic treatment need from clinical images. Seminars in Orthodontics 2021;27;2:164-171.

8. Koneru S, Tanikonda R. Salivaomics - A promising future in early diagnosis of dental diseases. Dent Res J (Isfahan) 2014;11(1):11-5.

9. Sunny S, Baby A, James BL, Balaji D, NVA, Rana MH, et al. A smart tele-cytology point-of-care platform for oral cancer screening. PLoS One 2019;14(11): e0224885.

10. Lin O. Telecytology for Rapid On-Site Evaluation: Current Status. J Am Soc Cytopathol 2018;7(1):1-6.

11. Hossain MM, Tasnim S, Sharma R, Sultana A, Shaik AF, Faizah F et al. Digital interventions for people living with non-communicable diseases in India: A systematic review of intervention studies and recommendations for future research and development. Digital Health 2019;5:2055207619896153.

12. Bhattacharya S, Hossain MM. National digital health blueprint of India: A need for implementation research. J Appl Sci Clin Pract 2020;1:21-2.

13. Yadav BK, Bihari C. Biobanking in India and its importance in cancer research. Edorium $\mathbf{J}$ Cancer 2017;3:10-12.

14. Joda T, Waltimo T, Probst-Hensch N, Pauli-Magnus C, Zitzmann NU. Health Data in Dentistry: An Attempt to Master the Digital Challenge. Public Health Genomics. 2019;22(1, 2):1-7.

15. Chhabra KG, Mulla SH, Deolia SG, Chhabra C, Singh J, Marwaha BS. Dental Informatics in India: Time to Embrace the Change. J Clin Diagn Res. 2016;10(3):ZE12-5.

16. Marya CM, Swati S, Nagpal R, Kataria S, Taneja P. Dental Informatics: Integrating Technology into 
Dentistry. Austin Dent Sci 2020;5(1):1025.

17. Mannocci A, Bontempi C, Giraldi G, Chiaradia G, de Waure C, Sferrazza A et al. EpiInfo as a research and teaching tool in epidemiology and statistics: strengths and weaknesses. Ig Sanita Pubbl. 2012;68(1):85-96. Italian.

18. Gohil R, Sharma S, Sachdeva S, Gupta S, Dhillon MS. Epi Collect 5: A Free, Fully Customizable Mobile-Based Application for Data Collection in Clinical Research.J Postgrad Med Edu Res 2020;12(2):1-4.

19. Dev, Monica, Singh B, Upendra, Khurana, Charu, et al. Analysis of Feedback to an Oral Health Promotion Website - 'eDant Seva' at a Tertiary Dental Institute in New Delhi. Available at :https://ssrn.com/abstract=3546124http://dx.doi.org/10.21 39/ssrn.3546124.

20. Sadoughi F, Erfannia L. Health Information System in a Cloud Computing Context. Stud Health Technol Inform. 2017;236:290-297.

21. Athavale AV, Zodpey SP. Public health informatics in India: the potential and the challenges. Indian journal of public health 2010;54(3):131.

22. Garg A, Rajkhowa MP, Dawar S. CollabDDS: ICT Tool for Advanced TeleRadiology. InProceedings of the 10th International Conference on Theory and Practice of Electronic Governance 2017;7:550-551.

23. Krishnan A, Nongkynrih B, Yadav K et al. Evaluation of computerized health management information system for primary health care in rural India. BMC Health Serv Res 2010;10:310. 